\title{
Studies on the Local Structure of Dilute 3d Transition Metal Ternary Dopants in $\mathrm{L1}_{2}$ Ordered $\mathrm{Ni}_{3} \mathrm{Al}$-Based Alloys
}

\author{
M. Balasubramanian, R. Lyver, J.I. Budnick and D.M. Pease \\ Physics Department and Institute of Materials Science, University of Connecticut, Storrs, \\ CT-06269, U.S.A.
}

\begin{abstract}
We have utilized the technique of XAFS to study the local environment of transition metal (TM) ternary dopants ( $\mathrm{Ti}, \mathrm{V}, \mathrm{Cr}, \mathrm{Mn}, \mathrm{Fe}$ and $\mathrm{Co}$ ) in $\mathrm{Ni}_{75} \mathrm{TM}_{\mathrm{X}} \mathrm{Al} 25-\mathrm{X}$. In $\gamma$ - $\mathrm{Ni} 3 \mathrm{Al}$, the first shell around $\mathrm{Al}$ consists of $12 \mathrm{Ni}$ atoms. $\mathrm{Ni}$ is surrounded by $8 \mathrm{Ni}$ and $4 \mathrm{AI}$ atoms. In all cases, the dopant K-edge XAFS results show only Ni backscattering contribution in the first peak of the Fourier transform. Al backscattering contribution is either completely absent or is so weak that it is masked by the strong dopant-Ni bond. The number of $\mathrm{Ni}$ near neighbors suggests that for $\mathrm{Ti}, \mathrm{V}, \mathrm{Cr}, \mathrm{Mn}$ and Fe absorbers, a majority of the dopant atoms occupy the Al sublattice. However, even in the case of Co dopants where studies by others indicate that the Co atoms occupy the $\mathrm{Ni}$ sublattice, no Al backscattering contribution can be detected. Also, the XANES of the various dopants exhibit a special feature at the edge, which appears to be due to p-d mixing.
\end{abstract}

\section{INTRODUCTION}

$\boldsymbol{\gamma}$-Ni3 Al is a well studied intermetallic alloy and has been used as a strengthening phase in Ni-based superalloys. It has been shown that addition of certain ternary elements can significantly enhance the ductility of $\mathrm{Ni} 3 \mathrm{Al}$ [1]. In order to understand the mechanism of improvement in the mechanical properties by ternary additives, it is necessary to know the site occupation and the local structural properties of the various ternary dopants in Ni3 Al. Well-ordered Ni3 $\mathrm{Al}$ takes the $\mathrm{L}_{2}$ ( $\mathrm{Cu}_{3} \mathrm{Au}$ prototype) crystal structure. This lattice has a face-centered-cubic cell where the cube corners are occupied by $\mathrm{Al}$ atoms and the face centers are occupied by $\mathrm{Ni}$ atoms. Hence, $\mathrm{Al}$ atoms are surrounded by $12 \mathrm{Ni}$ atoms and $\mathrm{Ni}$ atoms are surrounded by $8 \mathrm{Ni}$ and $4 \mathrm{Al}$ atoms in the first coordination shell. This ordered nature of the host alloy provides distinct possibilities by which the ternary additive can be distributed in the lattice. The dopant atoms may preferentially occupy either the Ni sublattice or the Al sublattice or may substitute randomly with no preference. In this investigation we have utilized the technique of XAFS to study the local structural and electronic properties of various $3 \mathrm{~d}$ transition metal (TM) dopants in the $\gamma$ - $\mathrm{Ni}_{3} \mathrm{Al}$ based host lattice.

\section{EXPERIMENTAL DETAILS}

Samples of $\mathrm{TM}_{2} \mathrm{Ni}_{75} \mathrm{Al}_{23}$ (TM: Ti, V, Cr, Mn, Fe and $\mathrm{Co}$ ) were prepared using a vacuum arc melter after argon backfill. Starting materials were $99.9 \%$ pure. One batch of the samples was initially given a homogenization anneal at $800^{\circ} \mathrm{C}$ for $\sim 100$ hrs and furnace cooled to room temperature. X-ray diffraction patterns of this batch of samples exhibited sharp peaks characteristic of the $\gamma-$ phase structure with good superlattice lines. In addition, a small amount of a second phase was also seen. In an attempt to obtain samples free of this contaminating second phase, another batch of samples was annealed at $800{ }^{\circ} \mathrm{C}$ for $\sim 12$ hrs and cooled by quenching in water. However, $x$-ray diffraction analysis again showed a small amount of second phase. The annealing treatment of the samples used in this study is presented in Table(1). The fraction of the second phase is of the order of a few percent and hence is not expected to affect the results of this study.

The XAFS measurements were performed at the National Synchrotron Light Source (NSLS) on beam line X-11 A using a Si (111) double-crystal monochromator. The monochromator was calibrated using appropriate pure metal foils. The incident beam intensity was monitored with an ionization chamber filled with an appropriate mixture of nitrogen gas and helium gas. The fluorescence signal from the dilute dopants was monitored using either the conventional fluorescence ion chamber or the Lytle detector filled with argon gas. Also, an appropriate filter made of a Z-1 material was used in conjunction with a soller slit assembly to reduce the elastic and Compton scattered background components. For Ti absorber no filter was used. Harmonic contamination was minimized by detuning the monochromator. All dopant $\mathrm{K}$-edge measurements were performed in the conventional fluorescence detection mode. Measurements at the $\mathrm{Fe}$ and $\mathrm{Co}$ edges did not show any unwanted Bragg peaks in the absorption spectra. Hence, these samples were measured by mounting them on a liquid nitrogen dewar. However, small Bragg peaks were seen in the spectra of other samples. Hence, these samples were spun during the final measurements to eliminate the deleterious effects of diffraction.

Data reduction was performed using programs based on the University of Washington XAFS analysis package. The background signal was subtracted from the spectra and the oscillatory portion of the data was isolated using standard procedures. The threshold energy was chosen at the inflection point in all cases. Quantitative analysis was performed by fitting the data to theoretical standards generated by FEFF [2]. The fitting was performed in r-space using FEFFIT, a fitting routine in the UWXAFS 3.0 package [3]. Care was taken to ensure that the number of parameters varied in the fitting procedure was always less than the number of independent data points in the data sets. 


\section{RESULTS AND DISCUSSION}

Figure (1a) shows the XAFS spectra for three representative absorbers ( $\mathrm{Co}, \mathrm{Fe}, \mathrm{Ti})$. It can be seen that the quality of the data is very good. It can be seen that the primary XAFS oscillations at the various edges are qualitatively similar. The XAFS spectra at the Fe edge is same as those presented by Pascarelli et al. [4] in a similar system. By analyzing the Fe XAFS in Fe-doped NizAl, Pascarelli et al [4]. concluded that to within the sensitivity of the technique, Fe dopants in these systems are coordinated only to Ni first near neighbors. The XAFS spectra shown in Figure (1a) are distinctly different from the XAFS spectra of Ni absorbers in $\gamma^{\prime}-$ Ni3Al as reported by Pascarelli et al [4]. This suggests that the absorbers represented in Figure (1a) do not have the same local structural order as $\mathrm{Ni}$ in $\mathrm{Ni} 3 \mathrm{Al}$.

\subsection{Analysis of XAFS data at the $\mathrm{Ti}, \mathrm{V}, \mathrm{Cr}, \mathrm{Mn}$ and Fe edges}

The data were weighted by $\mathbf{k}^{2}$ and Fourier transformed to obtain the radial distribution function of the various absorbers. The Fourier transform parameters used for the various dopants is given in Table (1). Representative Fourier transform at the Fe edge is illustrated in Figure (2). In the case of $\mathrm{Ti}, \mathrm{V}, \mathrm{Cr}, \mathrm{Mn}$ and $\mathrm{Fe}$ dopants initial qualitative analysis to determine the local order around the dopants was performed by allowing the dopants to occupy the Al sublattice. Hence, the standard for the first peak in the Fourier transform was taken to be $12 \mathrm{Ni}$ atoms at a distance corresponding to the undoped structure. The fit was performed by varying all four structural parameters, namely, the overall amplitude reduction factor $\mathrm{S}_{0}{ }^{2}$ (eff), disorder $\sigma^{2}$. distance $\mathrm{r}$, and the inner potential shift $\Delta \mathrm{E}_{0}$.

The structural parameters obtained in the case of $\mathrm{Ti}, \mathrm{V}, \mathrm{Cr}, \mathrm{Mn}$ and $\mathrm{Fe}$ are given in Table (1). A representative fit to data at the Fe edge is shown in Figure (1b). It can be seen that the fit is in good agreement with the data. Hence, it can be seen that the first peak in the Fourier transform can be well represented by assuming only Ni backscattering. In order to test for the presence of Al first near neighbors an Al backscattering contribution was also added. Detailed fitting analysis with various constraints, $k$ weighting, and k- space window widths was performed. However, inclusion of Al backscattering did not improve the quality of the fit. Hence, we come to the conclusion that in the case of $\mathrm{Ti}, \mathrm{V}, \mathrm{Cr}, \mathrm{Mn}$ and $\mathrm{Fe}$ dopants the first shell around the dopants can be well represented by dopant-Ni bonds. No evidence for the presence of Al first near neighbors can be seen in the XAFS.

In the case of single scattering, $\mathrm{S}_{0}{ }^{2}$ (eff) depends on the many-body amplitude reduction factor $\mathrm{S}_{0}{ }^{2}$ and the number of backscatterers. $\mathrm{S}_{0}{ }^{2}$ should depend only on the central absorber and so is transferable to any system with the same central atom. Hence, by assuming $S_{0}{ }^{2}$ of the system under investigation to be equal to that of a well known reference (such as a pure metal foil) the number of near neighbors in the shell can be determined. The values for $\mathrm{S}_{0}{ }^{2}$ of $3 \mathrm{~d}$ transition metals are typically between 0.70 and 0.85 and hence in the case of $\mathrm{Ti}, \mathrm{V}, \mathrm{Cr}, \mathrm{Mn}$ and $\mathrm{Fe}$ absorbers the majority of the dopant atoms occupy the Al sublattice. In the case of Fe dopants, by using the $S_{0}^{2}$ value of metallic $\mathrm{Fe}$, we obtain a value of $10.75(60) \mathrm{Ni}$ atoms in the first coordination shell. This is consistent with the results of Pascarelli et al. [4], who find that $\mathrm{Fe}$ in a similar system is coordinated to 11 (1) $\mathrm{Ni}$ atoms.

\subsection{Analysis of the XAFS data at the Co edge}

The XAFS of Co absorbers is shown in Figure (1a). Earlier studies by others, both experimental and theoretical calculations, have clearly indicated that in Co-doped $\mathrm{Ni}_{3} \mathrm{Al}$ the $\mathrm{Co}$ atoms almost exclusively occupy the Ni sublattice $[5,6]$. Comparison of the Co XAFS in Figure (1a) with the Ni XAFS in Ni3Al reported by Pascarelli et al. [4] reveals that they are distinctly different. Particularly, the major difference between the two spectra are seen for low-k values. The influence of Al backscattering will be very strong for low-k values. This indicates that the local order around the $\mathrm{Co}$ atoms is not similar to that of $\mathrm{Ni}$ atoms in $\mathrm{Ni3} \mathrm{Al}$. The $\mathbf{k}^{2}$ weighted Fourier transform of Co dopants is shown in Figure (lb).
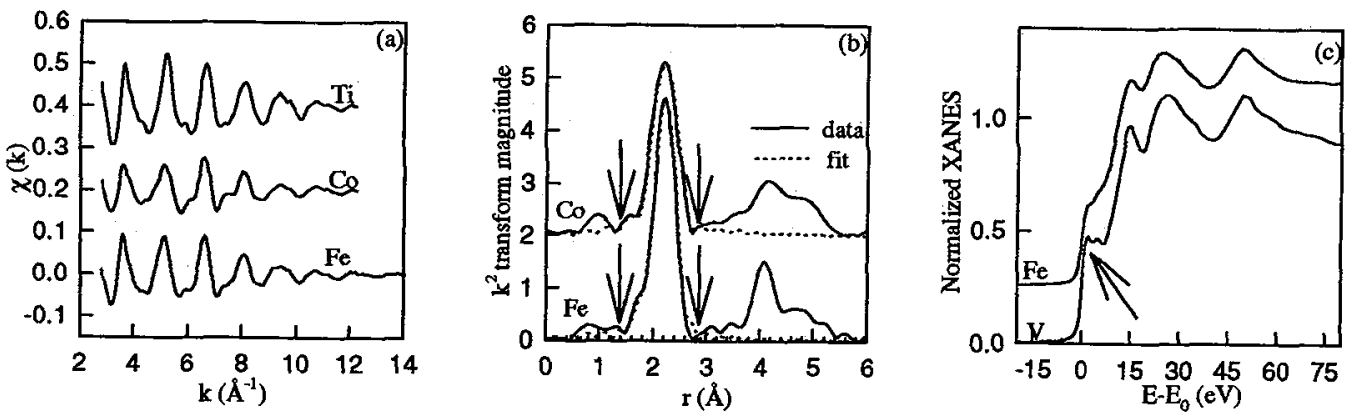

Figure 1: (a) Dopant $\mathrm{K}$-edge XAFS spectra taken at room temperature, (b) $\mathrm{K}^{2}$ weighted Fourier transform and corresponding fits. The fit range is indicated by the arrows, (c) XANES spectra at the dopant $\mathrm{K}$-edge.

As earlier studies by others $[5,6]$ have indicated that Co dopants occupy Ni-type sites, the standards to model the first peak of the Fourier transform were generated from FEFF by substituting Co in the Ni3Al lattice and allowing the Co dopants to occupy the Ni-type sites. In such a site, Co will be coordinated to $8 \mathrm{Ni}$ atoms and $4 \mathrm{Al}$ atoms. Further, from the qualitative comparisons in the previous paragraph it appears that the Al backscattering contribution may be weak. Hence, the initial fit was performed by 
introducing only a Co-Ni single scattering path (i.e., the standard corresponded to that generated by letting Co occupy the Ni sublattice, and taking only the $\mathrm{Co}-\mathrm{Ni}$ contribution). The Fourier transform parameters used in the fitting procedure are presented in Table (1). All four structural parameters of the $\mathrm{Co}-\mathrm{Ni}$ shell, the overall amplitude reduction factor $\mathrm{S}_{0}{ }^{2}$ (eff), distance r, disorder $\sigma^{2}$ and inner potential shift $\Delta \mathrm{E}_{0}$, were allowed to vary in the fit. Figure (1b) illustrates the fits obtained assuming that the first peak in the Fourier transform can be represented by $8 \mathrm{Ni}$ neighbors. It can be seen that the fit is in very good agreement with the experimental data. The XAFS structural parameters are reported in Table (1). Using the $\mathrm{S}_{0}{ }^{2}$ value of Co foil, we find that the Co absorbers are coordinated to $8.20(70) \mathrm{Ni}$ atoms in the first shell. Just as in the case of the previous dopants, an attempt to see any Al backscattering contribution was carried out; however, again the fits revealed that $\mathrm{Al}$ backscattering contribution was either absent or too weak to be detected within the sensitivity of the measurements.

In summation, XAFS measurements at the K-edges of the dilute $3 \mathrm{~d}$ dopants reveal that the first coordination shell around the dopant atoms can be well represented by dopant-Ni bonds. Al backscattering contribution is either completely absent or is so weak that it is masked by the strong dopant-Ni bonds. The number of $\mathrm{Ni}$ first near neighbors indicate that in the case of $\mathrm{Ti}$, $\mathrm{V}$, $\mathrm{Cr}$, $\mathrm{Mn}$ and Fe absorbers a majority of the dopant atoms occupy the Al sublattice. However, in the case of Co absorbers our measurements indicate that $\mathrm{Co}$ is coordinated to $\sim 8 \mathrm{Ni}$ atoms and no $\mathrm{Al}$ atoms in the first shell. The presence of $8 \mathrm{Ni}$ atoms will be consistent with Ni sublattice occupancy, if we can account for the absence of $4 \mathrm{Al}$ neighbors. A model based on a highly distorted Al sublattice gives a possible explanation for the absence of these bonds: Detailed XAFS data analysis and a possible model to account for the absence of the Al backscattering contributions will be subsequently presented elsewhere.

Table (1): Summary of the annealing treatment conditions, $k$-space and r-space window widths used in the XAFS analysis and XAFS structural parameters obtained by fitting to standards generated from the ab initio calculations based on FEFF. The first peak in the Fourier transform can be well represented by dopant-Ni bonds. The structural parameters of the first shell of Ni neighbors, the overall amplitude factor, the disorder, the inner potential shift and the near neighbor distance were all varied. In the case of $\mathrm{Fe}$ and $\mathrm{Co}$ absorbers the temperature-independent factors, the inner potential shift and the overall amplitude factor, were constrained to be the same. The numbers in the parenthesis are the uncertainties of the last digit(s). Samples were either annealed for $\sim 100$ hours at $800^{\circ} \mathrm{C}$ and furnace cooled (FC) or they were annealed for $\sim 12$ hours at $800^{\circ} \mathrm{c}$ and cooled by quenching in ice cold water (QC).

$\begin{array}{lclcccrr}\text { System } & \begin{array}{c}\text { Annealing } \\ \text { Condition }\end{array} & \Delta \mathrm{k}\left(\AA^{-1}\right) & \Delta \mathrm{r}(\AA) & \text { Temperature } & \begin{array}{c}\text { Assumed No. of } \\ \text { neighbors }\end{array} & S_{0}^{2} \text { (eff) } & \mathrm{r}(\AA) \\ \mathrm{Ti}_{2} \mathrm{Ni}_{75} \mathrm{Al}_{23} & \text { QC } & 2.7-11.75 & 1.50-2.90 & \sim 296 \mathrm{~K} & 12 & 0.72(7) & 2.534(7) \\ \mathrm{V}_{2} \mathrm{Ni}_{75} \mathrm{Al}_{23} & \text { FC } & 3.0-13.5 & 1.50-2.80 & -296 \mathrm{~K} & 12 & 0.76(7) & 2.495(6) \\ \mathrm{Cr}_{2} \mathrm{Ni}_{75} \mathrm{Al}_{23} & \text { QC } & 3.0-12.5 & 1.50-2.80 & -296 \mathrm{~K} & 12 & 0.71(6) & 2.482(6) \\ \mathrm{Mn}_{2} \mathrm{Ni}_{75} \mathrm{Al}_{23} & \text { QC } & 3.0-12.5 & 1.50-2.80 & \sim 296 \mathrm{~K} & 12 & 0.64(5) & 2.519(5) \\ \mathrm{Fe}_{2} \mathrm{Ni}_{75} \mathrm{Al}_{23} & \text { FC } & 3.0-14.0 & 1.50-2.80 & -296 \mathrm{~K} & 12 & 0.69(4) & 2.501(4) \\ \mathrm{Fe}_{2} \mathrm{Ni} 75 \mathrm{Al}_{23} & \text { FC } & 3.0-14.0 & 1.50-2.80 & \sim 80 \mathrm{~K} & 12 & 0.69(4) & 2.499(3) \\ \mathrm{Co}_{2} \mathrm{Ni}_{75} \mathrm{Al}_{23} & \text { FC } & 2.75-11.5 & 1.50-2.80 & -296 \mathrm{~K} & 8 & 0.76(6) & 2.495(4) \\ \mathrm{Co}_{2} \mathrm{Ni}_{75} \mathrm{Al}_{23} & \text { FC } & 2.75-11.5 & 1.50-2.80 & -80 \mathrm{~K} & 8 & 0.76(6) & 2.491(4)\end{array}$

\subsection{XANES}

The near edge spectra of $\mathrm{V}$ and $\mathrm{Fe}$ absorbers is shown in Figure (1c). The energy origin is chosen at the inflection point. We note that a special feature, split into a well resolved doublet is present for $\mathrm{V}$ absorbers (indicated by the arrow). A similar feature is also seen for $\mathrm{Ti}$ and $\mathrm{Cr}$ absorbers as well. The feature is not as prominent for $\mathrm{Mn}$ and $\mathrm{Fe}$ absorbers and might be present as a small shoulder at the edge. XAFS analysis indicates that the majority of these dopants occupy the Al sublattice. The feature seems to decrease in intensity as the d-hole count decreases. The presence of this feature might be due to transitions into the metal d-like states. As the experiment was performed at the $\mathrm{K}$-edges of the various dopants, dipole selection rules permit transitions from 1 s states to empty $p$-like states. The presence of this feature might then indicate the mixing of $p$ and $d$ states. Transition into empty $p$ parts of the mixed p-d states will be permitted. It is interesting to see such mixing in systems with cubic symmetry. But it should be noted that the distortions around the dopant atom could slightly distort the local symmetry, allowing for the mixing. Further investigation to understand the presence of this unique feature is currently underway.

We acknowledge support by the U.S. DOE under contract No. DE-FG05-89-ER45384.

\section{References}

[1] See for example, G. Sauthoff, Intermetallics (VCH, Weinheim, 1995).

[2] J. Mustre de Leon, J. J. Rehr, S. I. Zabinsky, and R. C. Albers, Phys. Rev. B 4 (1991) 4146- 4153.

[3] E. A. Stem, M. Newville, B. Ravel, Y. Yacoby, and D. Haskel, Physica B 208 \& 209, (1995) 117-118.

[4] S. Pascarelli, F. Boscherini, S. Mobilio, K. Lawniczak-Jablonska, and R. Kozubski, Phys. Rev. B 49 (1994), 14984-14990.

[5] D. Shindo, M. Kikuchi, M. Hirabayashi, S. Hanada, and O. Izumi, Trans. Jpn. Inst. Met. 29 (1988) 956-961.

[6] M. H. F. Sluiter and Y. Kawazoe, Phys. Rev. B 51 (1995) 4062-4073. 